\title{
Germline PALB2 Variants and PARP Inhibitors in Endometrial Cancer
}

\author{
Michael A. Cilento, MBBS ${ }^{1,2}$; Nicola K. Poplawski, MD,4; Sellvakumaram Paramasivam, MBBS ${ }^{5}$; \\ David M. Thomas, MBBS, PhD ${ }^{6}$; and Ganessan Kichenadasse, MBBS ${ }^{1,7}$
}

\begin{abstract}
PARP inhibitors are orally administered antineoplastic agents that affect the homologous recombination (HR) repair pathway, and are approved by the FDA for the treatment of ovarian, breast, pancreatic, and prostate cancers. This report presents a case of recurrent endometrial carcinoma occurring in a woman with a germline pathogenic PALB2 whole-exon deletion. This uncommon finding in a patient with endometrial carcinoma provided the opportunity to use a management strategy of PARP inhibition with olaparib, resulting in a prolonged response to treatment; however, disease progression eventually occurred. Further studies are required to elucidate the mechanisms underlying resistance to PARP inhibition, and the potential future treatment options in this setting. Current recommendations for risk management of female carriers of PALB2 variants focus on breast and ovarian cancer risk. This case raises the additional question of a potential role for risk-reducing hysterectomy in female carriers of $P A L B 2$ variants.
\end{abstract}

J Natl Compr Canc Netw 2021;19(11):1212-1217 doi: $10.6004 /$ jnccn.2021.7067

\footnotetext{
${ }^{1}$ Department of Medical Oncology, Flinders Medical Centre, Adelaide, SA; ${ }^{2}$ Faculty of Health and Medical Sciences, The University of Adelaide, Adelaide, SA; ${ }^{3}$ Adult Genetics Unit, Royal Adelaide Hospital, Adelaide, SA; ${ }^{4}$ Adelaide Medical School, University of Adelaide, Adelaide, SA; ${ }^{5}$ Gynaecological Oncology, FlindersMedical Centre, Adelaide, SA; ${ }^{6}$ The Kinghorn Cancer Centre, Garvan Institute of Medical Research, Darlinghurst, NSW; and

${ }^{7}$ Flinders Centre for Innovation in Cancer, Flinders University, Adelaide, SA, Australia.
}

PARP inhibitors are orally administered antineoplastic agents that affect the homologous recombination (HR) repair pathway, and are FDA-approved for the treatment of ovarian, breast, pancreatic, and prostate cancers. The HR repair pathway is one mechanism responsible for repair of double-strand DNA breaks, with BRCAl and BRCA2 proteins playing a key role in this process. ${ }^{1}$ The Partner and Localizer of BRCA2 (PALB2) protein has emerged as an important BRCA2-interacting protein and is encoded by the PALB2 gene.

The PALB2 gene is located on chromosome $16 \mathrm{p} 12.2$ and comprises 13 exons, which encode a 1,186-amino acid protein. This protein plays an important role in genome integrity maintenance primarily through its mediation of the HR repair pathway. Fanconi anemia (FA) subtype FA-N (MIM 610832) is the consequence of biallelic variants in $P A L B 2$, whereas monoallelic variants are known to predispose to solid tumors, including breast, ovarian, and pancreatic cancers (MIM 114480). ${ }^{2}$

Patients with germline BRCA1, BRCA2, or PALB2 pathogenic or likely pathogenic variants in whom there is somatic loss of the second wild-type allele can develop cancers. In these patients, the usual double-strand DNA repair pathway is impaired, and they are referred to as being HRdeficient (HRD). In the setting of HRD, there is an intrinsic reliance upon single-strand DNA repair pathways that include the mismatch repair (MMR) pathway, nucleotide excision repair, and the base excision repair (BER) pathway.

The BER pathway works to address single-strand DNA damage from alkylation, oxidation, and reactive oxygen species. BER is mediated in part by PARP, with interruption of this pathway ultimately leading to double-strand breaks. ${ }^{3}$ The use of PARP inhibitors to block BER in tumors with HRD results in the accumulation of double-strand breaks and ultimately cell death, the socalled therapeutic synthetic lethality.

This report presents a case of recurrent endometrial carcinoma occurring in a patient with a germline pathogenic $P A L B 2$ variant who had an exceptional response to the PARP inhibitor olaparib.

\section{Case Report}

A 58-year-old woman presented to her primary care physician in October 2014 with symptoms of urinary 
incontinence. A large pelvic mass was detected on clinical examination. Imaging with $\mathrm{CT}$ scans revealed abnormal thickening of the endometrial lining, extensive peritoneal soft tissue nodules, and a soft tissue mass in the anterior pelvis measuring $23 \times 21 \times 104$ $\mathrm{mm}$ consistent with omental caking. CA-125 serum tumor marker at time of presentation was elevated at $1,155 \mathrm{kU} / \mathrm{L}$ (normal range, $<35 \mathrm{kU} / \mathrm{L}$ ).

The patient underwent primary surgery with radical hysterectomy, bilateral salpingo-oophorectomy, lymph node dissection, and removal of tumor from the diaphragm and Gerota fascia. There was no evidence of disease on postoperative imaging. Surgical pathology showed a high-grade serous carcinoma of endometrial origin with the bulk of the tumor arising from the lower fundus, lower uterine segment, and extending to cervical stroma, measuring $40 \mathrm{~mm}$ in greatest extent. Although most of the primary tumor was confined to the endometrium, in areas there was smooth muscle extending in between islands of tumor cells and compression of the underlying myometrium by the tumor mass that showed a broad pushing margin; the serosa was intact. There were metastases to fallopian tubes, tumor seen as serosal surface nodules on both ovaries, and extensive involvement of the peritoneum. All resected lymph nodes were negative; there was no evidence of lymphovascular invasion. However, peritoneal washings were positive for malignant cells. The tumor was MMR-proficient with normal expression of MLH1, PMS2, MSH2, and MSH6 by immunohistochemistry. Additional testing for p53 protein staining and molecular testing for POLE was not performed, given the characteristic pathologic findings at the time of surgery; however, the utility of these complementary tests in endometrial cancer has since been described, with most endometrial serous carcinomas now known to display p53 abnormalities. ${ }^{4}$

Surgery was followed by postoperative chemotherapy with 6 cycles of carboplatin and paclitaxel, with normalization of CA-125 level to $6 \mathrm{kU} / \mathrm{L}$. Surveillance was then undertaken with regular clinical assessment, monitoring of CA-125 tumor marker, and CT imaging. In September 2016, the CA-125 was elevated at $94 \mathrm{kU} / \mathrm{L}$, and repeat imaging was performed with FDG-PET/CT scan (Figure 1). There was evidence of an FDG-avid, 2.5-cm aortocaval nodal mass (standardized uptake value [SUV], 6.1) and 1 additional mild to moderate focus of FDG activity in the left posterior pelvic floor (SUV, 4.16), with no other sites of disease. The patient began carboplatin and pegylated liposomal doxorubicin chemotherapy. After completion of 5 cycles a complete response of the para-aortic lymphadenopathy and pelvic floor node was seen, and the patient went on to further clinical surveillance from March 2017 onwards. Progressive disease was detected in July 2018 and the patient received further chemotherapy with carboplatin and gemcitabine, completing 6 cycles by December 2018 with a partial response.

In April 2019, recurrent disease was again detected on CT imaging with a large mass in the left pelvis measuring $72 \times 63 \mathrm{~mm}$ (Figure 2), along with disease adjacent to the splenic hilum. Left hydronephrosis and hydroureter was present, and her CA-125 level was elevated at 1,407 kU/L.

Next-generation sequencing (NGS) for a wide panel of genomic variants was performed on DNA from tumor tissue that showed a low tumor mutation burden, stable microsatellite status, and loss of exon 11 of PALB2. Multiplex ligation-dependent probe amplification (MLPA) in DNA extracted from lymphocytes confirmed germline deletion of the entire PALB2 exon 11 coding sequence, and part of the adjoining intron 10 and intron 11 sequence (NM_024675.3(PALB2):c.(3113 + 1_3114-1)_(3201 + 1_3202-1) del). The variant is considered pathogenic using American College of Medical Genetics and Genomics (ACMG) variant classification guidelines. ${ }^{5}$ The patient initiated the PARP inhibitor olaparib and experienced only mild toxicity with anemia and fatigue. Interval imaging in July 2019 and February 2020 (Figure 3) showed an exceptional response in the known sites of disease with the pelvic mass decreasing to just $26 \times 18 \mathrm{~mm}$, and CA125 level decreasing from $1,407 \mathrm{kU} / \mathrm{L}$ to a nadir level of $15 \mathrm{kU} / \mathrm{L}$. The patient developed biochemical and radiographic progression 23 months later.

The family history relevant to this case was from the maternal side, with no relevant cancer diagnoses on the paternal side. Maternal family history included the patient's mother having had an endometrial tumor of unknown type at age 31 years and also 2 synchronous colorectal cancers; an uncle with primary lung cancer at age 55 years; a grandmother with an endometrial tumor at age 34 years; and a great-grandmother with breast cancer at unknown age. The patient was referred to her local clinical genetics service to discuss predictive genetic testing. Although the patient does not have any genetic siblings, she does have 3 children for whom predictive testing may guide decisions on cancer screening and risk-reducing procedures.

\section{Discussion}

Endometrial carcinoma is the most common malignancy of the female genital tract in the United States. It is estimated in 2021 that 66,570 women in the United States will be diagnosed with endometrial carcinoma, which is expected to result in 12,940 deaths based on the American Cancer Society's predictions. ${ }^{6}$

Although most endometrial carcinoma occurs because of sporadic somatic mutational events, approximately $5 \%$ of cases occur in patients with a germline cancer predisposing variant, most commonly a germline variant in an MMR gene (Lynch syndrome; MIM 120435). ${ }^{7}$ Screening for germline variants in patients with endometrial 


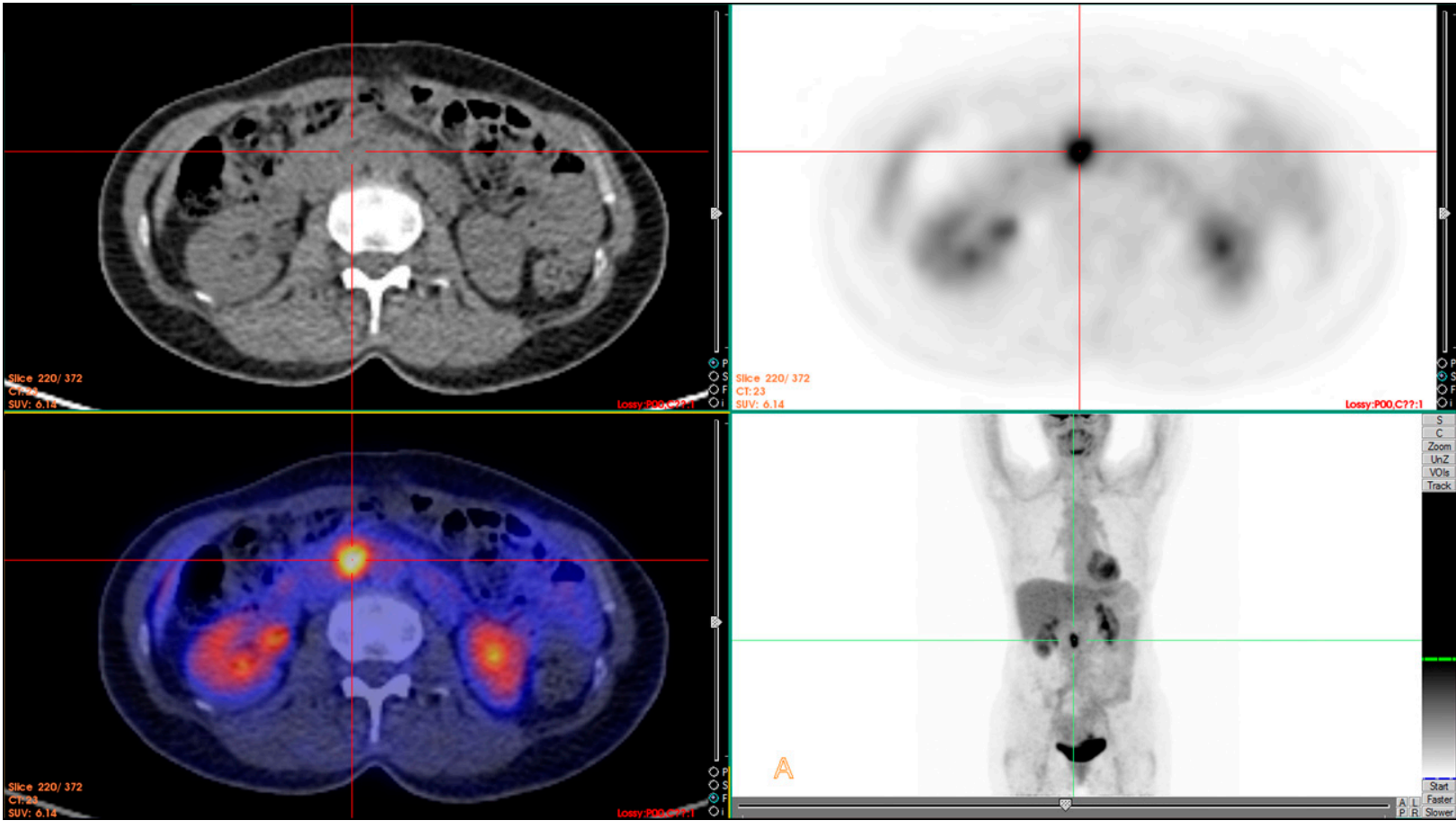

Figure 1. PET/CT imaging showing intense FDG-avid aortocaval nodal mass at time of first recurrence.

carcinoma has helped to define the frequency of different germline variants in cancer predisposing genes in these patients, with a recent series of women from China identifying 1 patient with a pathogenic variant in the PALB2 gene. ${ }^{8}$ With the increasing use of molecular panel testing for solid

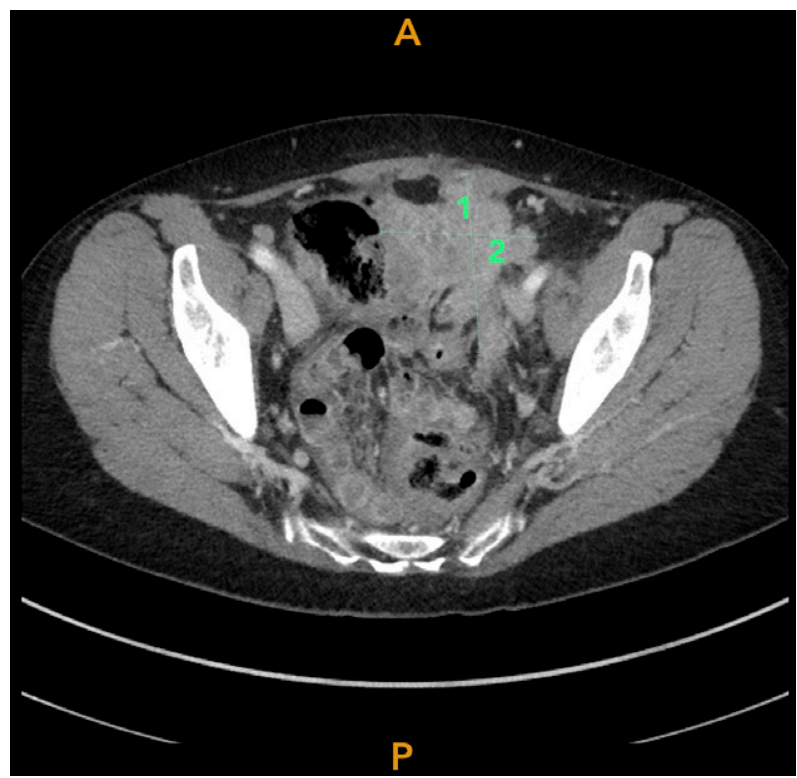

Figure 2. CT imaging of pelvis showing progressive disease after third-line chemotherapy, with left pelvis mass measuring $72 \mathrm{~mm}$ in longest diameter (1) $\times 63 \mathrm{~mm}$ in perpendicular plane (2). Abbreviations: $A$, anterior; $P$, posterior. tumors, genomic variants are readily detected that have also been described with germline inheritance. There is an increasing reliance on surrogate markers such as variant allelic frequency or concurrent germline testing to identify hereditary mutations, which in turn can trigger predictive testing of relatives when a pathogenic familial cancer gene variant is detected. The integration of somatic and germline variant detection is a critical step; loss of heterozygosity $(\mathrm{LOH})$ analysis can uncover heterozygous germline variants that are under potential selection in the tumor. $\mathrm{Lu}$ et $\mathrm{al}^{9}$ previously described the patterns of germline variants across 12 cancer types, and after $\mathrm{LOH}$ analysis testing of an expanded list of 34 genes (known and likely oncogenes excluded), a total of 7 genes (BRCA1, BRCA2, RAD51D, PALB2, RAD51C, ATM, and BRIP1) were found to be significant. With all of these genes known to play a role in the HR pathway, there is the potential for these findings to direct translational work with PARP inhibitors across a wider range of tumor types.

The increasingly detailed description of the broad landscape of genomic variants in uterine carcinoma is now beginning to inform clinical practice not only for investigators, but also for community medical oncologists, for whom use of NGS tumor testing has now become commonplace. Endometrioid endometrial carcinomas are known to have a high frequency of microsatellite instability and POLE mutations predicting benefit to immune checkpoint blockade, ${ }^{10}$ although rare but important biomarkers such as NTRK gene fusion-positivity allows patients 


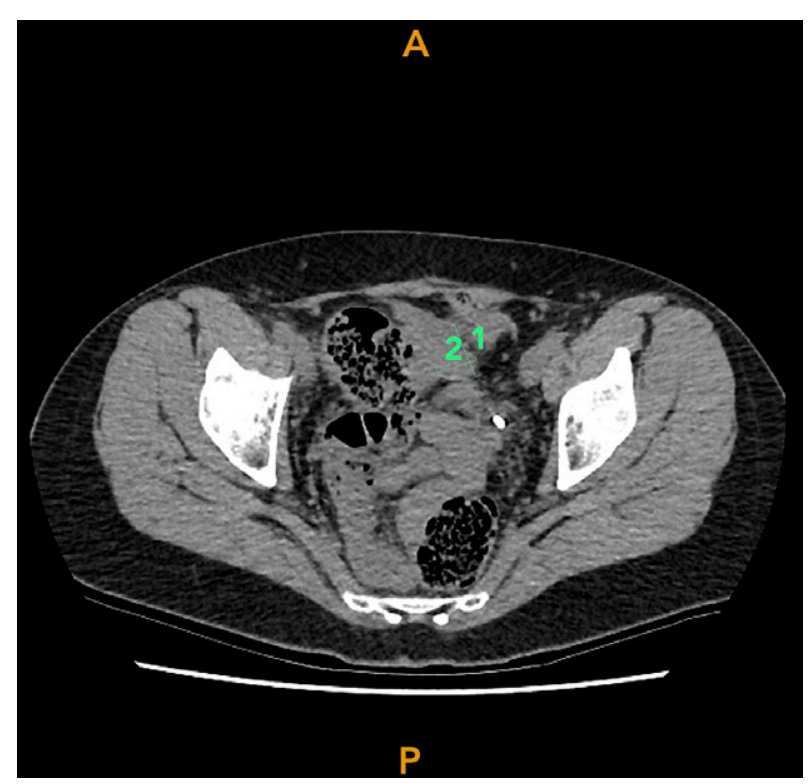

Figure 3. CT imaging of pelvis after 10 months of olaparib, showing response in pelvic mass decreasing to $26 \mathrm{~mm}$ in longest diameter (1) $\times 18 \mathrm{~mm}$ in perpendicular plane (2).

Abbreviations: $A$, anterior; $P$, posterior.

another targeted treatment option in the second line. Uterine serous carcinomas have been described as sharing characteristics with high-grade serous ovarian cancer, but also basal-like breast cancers. There is a high frequency of alterations in the PI(3)K/AKT pathway in uterine serous carcinoma, with mutations in PIK3CA occurring in $42 \%$ of these patients; further opportunities for translational targeted therapy certainly exist. ${ }^{11}$ Gene alterations affecting the HR repair pathway have also now become a therapeutic target across cancer types.

The finding of a germline PALB2 variant in our patient with serous endometrial carcinoma is uncommon. Molecular alterations in $P A L B 2$ occur in $1.82 \%$ of all primary cancers, with lung adenocarcinoma, colon adenocarcinoma, breast invasive ductal carcinoma, and endometrial endometrioid adenocarcinoma having the greatest prevalence (Figure 4). ${ }^{12}$ The most common alterations in the PALB2 gene are point mutations. Reports of the frequency of alterations in PALB2 range from $4.49 \%$ of endometrial carcinomas in the GENIE database, ${ }^{12}$ to as high as $8.49 \%$ of endometrial cancers regardless of histologic type in The Cancer Genome Atlas Uterine Corpus Endometrial Carcinoma dataset. ${ }^{10}$

This uncommon finding of a pathogenic PALB2 whole-exon deletion in a patient with high-grade serous endometrial carcinoma provided the opportunity to use a PARP inhibitor like olaparib, for which there is a strong scientific rationale extrapolated from other tumor types with HRD, such as BRCA-mutated ovarian cancer. BRCA1 and $B R C A 2$ germline variants have been recognized as cancer predisposition genes for some time now, with literature beginning to describe the frequency of different cancers in these individuals. In a recent meta-analysis of 13,871 carriers of known germline BRCA1 and BRCA2 mutations undergoing risk-reducing salpingo-oophorectomy, the prevalence of endometrial cancer was just $0.59 \%$ and $0.16 \%$, respectively. ${ }^{13}$ Unfortunately there remains a paucity of data for carriers of PALB2 variants, in terms of the frequency of different cancers and their expected clinical course, making it difficult to counsel patients about their individual risk.

Olaparib was the PARP inhibitor used for this patient; however, many other PARP inhibitors are now also approved across different tumor types, including rucaparib, niraparib, veliparib, and talazoparib.

Our patient had a prolonged response to platinum chemotherapy, and it has previously been shown that PARP inhibitor response is closely related to platinum sensitivity in other tumor types, such as ovarian carcinoma. ${ }^{14}$ Although our patient had a prolonged response to PARP inhibition, she eventually developed resistant disease. PARP inhibitor resistance mechanisms in PALB2-mutated cancers are poorly understood. Although the mechanisms of resistance require further research, studies from $B R C A$-mutated cancers indicate that HR repair restoration is one major contributing event. ${ }^{15,16}$ In high-grade serous ovarian carcinomas, some authors have suggested that one of the PARP inhibitor resistance mechanisms may be reduction in levels of poly(ADP-ribose) glycohydrolase (PARG), which would be expected to preserve a residual PARP1-initiated DNA damage response to DNA single-strand breaks. ${ }^{17}$ Whether similar mechanisms of resistance are in action in the setting of PARP inhibition for PALB2-mutated cancers requires further investigation. Understanding the mechanisms of resistance will also help to inform the utility, if any, of switching between PARP inhibitors at progression, an issue for which there are currently limited data.

Determination that the PALB2 variant was of germline origin also provided our patient's genetic relatives the opportunity to consider predictive testing for the variant to inform their cancer risk, recommended cancer surveillance and risk-reducing strategies, and reproductive decisions. Current recommendations for risk management of female carriers of $P A L B 2$ variants focus on breast and ovarian cancer risk. ${ }^{18}$ The focus of these recommendations is based on the estimated lifetime risks of these cancers and their association with germline $P A L B 2$ variants; the risk to 80 years was $53 \%$ for female breast cancer, $5 \%$ for ovarian cancer, and $2 \%$ to $3 \%$ for pancreatic cancer. ${ }^{19}$ This case raises the additional question of a potential role for risk-reducing hysterectomy in female carriers of PALB2 variants.

\section{Conclusions}

Our case of a patient with high-grade serous endometrial carcinoma with a prolonged response to platinum 


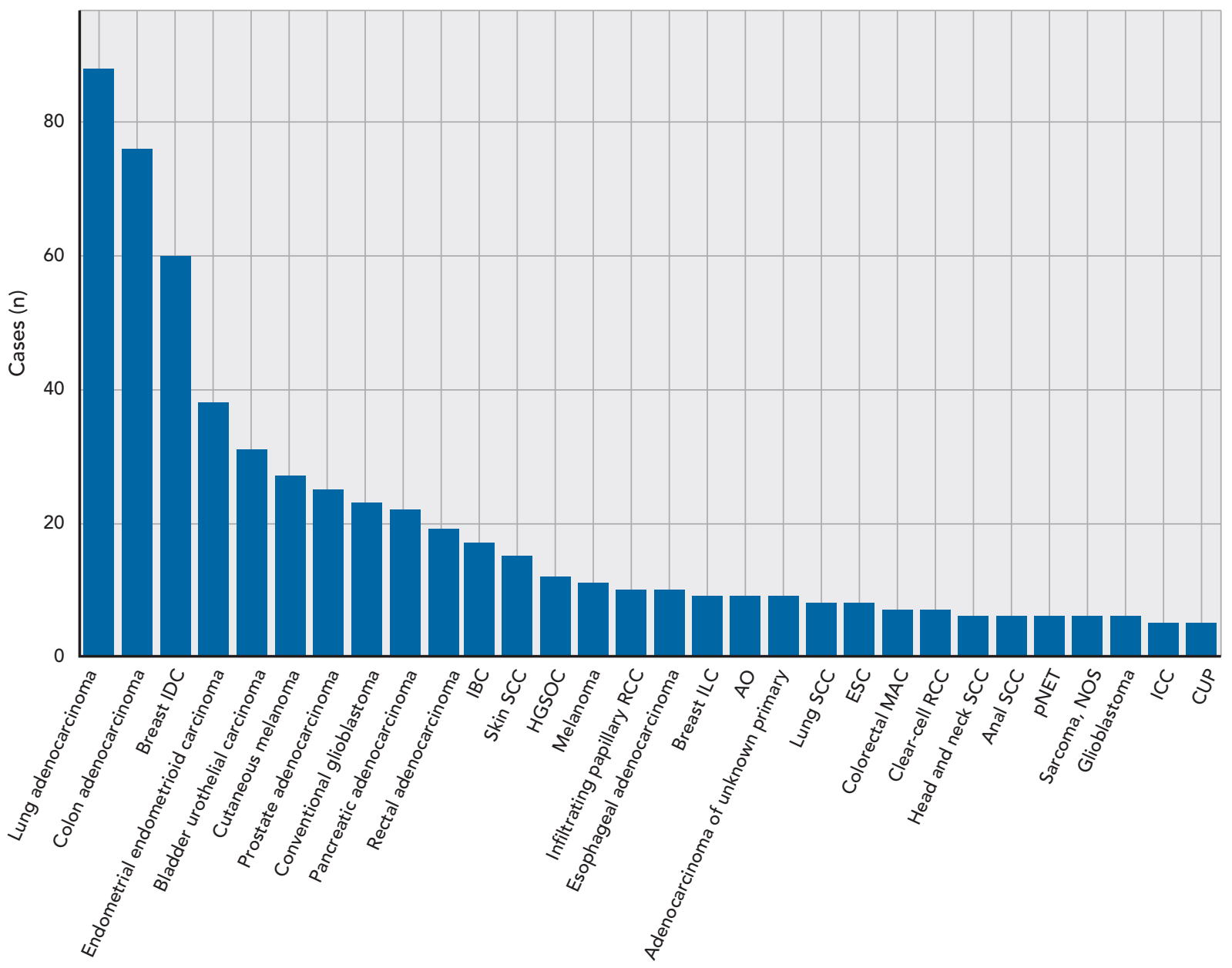

Figure 4. Distribution of molecular alteration in PALB2 across different primary tumors in the AACR Project GENIE database.

Abbreviations: AACR, American Association for Cancer Research; AO, anaplastic oligodendroglioma; CUP, cancer of unknown primary; ESC, endometrial serious adenocarcinoma; HGSOC, high-grade serous ovarian carcinoma; IBC, invasive breast carcinoma; ICC, intrahepatic cholangiocarcinoma; IDC, invasive ductal carcinoma; ILC, invasive lobular carcinoma; MAC, mucinous carcinoma; NOS, not otherwise specified; pNET, pancreatic neuroendocrine tumor; RCC, renal cell carcinoma; SCC, squamous cell carcinoma.

chemotherapy, followed by impressive response to PARP inhibition in the setting of a germline PALB2 variant, highlights the importance of testing for genetic HR pathway variants, even for uterine primaries. Further studies are required to elucidate the mechanisms underlying resistance to PARP inhibition, and the potential future treatment options in this setting.

There is a need to ensure that genetic testing is applied widely over a broad range of tumor types, not only for the benefit of patients for whom the result may directly inform their cancer management but also for their families in whom appropriate institution of timely genetic counseling has the potential to saves lives.

Submitted January 11, 2021; final revision received June 8, 2021; accepted for publication June 8, 2021.

Disclosures: The authors have disclosed that they have not received any financial consideration from any person or organization to support the preparation, analysis, results, or discussion of this article.

Correspondence: Michael A. Cilento, MBBS, Department of Medical Oncology, Flinders Medical Centre, Flinders Drive, Bedford Park, SA 5042, Australia. Email: michael.cilento@sa.gov.au

\section{References}

1. Lord CJ, Ashworth A. BRCAness revisited. Nat Rev Cancer 2016;16: 110-120.

2. Ducy M, Sesma-Sanz L, Guitton-Sert L, et al. The tumor suppressor PALB2: inside out. Trends Biochem Sci 2019;44: 226-240.
3. Bryant HE, Schultz N, Thomas HD, et al. Specific killing of BRCA2-deficient tumours with inhibitors of poly(ADP-ribose) polymerase. Nature 2005;434:913-917.

4. Hoang LN, Kinloch MA, Leo JM, et al. Interobserver agreement in endometrial carcinoma histotype diagnosis varies depending on the cancer 
genome atlas (TCGA)-based molecular subgroup. Am J Surg Pathol 2017:41:245-252.

5. Richards S, Aziz N, Bale S, et al. Standards and guidelines for the interpretation of sequence variants: a joint consensus recommendation of the American College of Medical Genetics and Genomics and the Association for Molecular Pathology. Genet Med 2015;17:405-424.

6. Siegel RL, Miller KD, Fuchs HE, et al. Cancer statistics, 2021. CA Cancer J Clin 2021;71:7-33.

7. Resnick KE, Hampel $\mathrm{H}$, Fishel $\mathrm{R}$, et al. Current and emerging trends in Lynch syndrome identification in women with endometrial cancer. Gynecol Oncol 2009;114:128-134.

8. Tian $W, B i R$, Ren $Y$, et al. Screening for hereditary cancers in patients with endometrial cancer reveals a high frequency of germline mutations in cancer predisposition genes. Int J Cancer 2019;145:1290-1298.

9. Lu C, Xie M, Wendl MC, et al. Patterns and functional implications of rare germline variants across 12 cancer types. Nat Commun 2015;6:10086.

10. Cancer Genome Atlas Research Network, Kandoth C, Schultz N, et al. Integrated genomic characterization of endometrial carcinoma. Nature 2013;497:67-73.

11. Le Gallo M, O'Hara AJ, Rudd ML, et al. Exome sequencing of serous endometrial tumors identifies recurrent somatic mutations in chromatin-remodeling and ubiquitin ligase complex genes. Nat Genet 2012;44:1310-1315.
12. AACR Project GENIE Consortium. AACR Project GENIE: powering precision medicine through an international consortium. Cancer Discov 2017; 7:818-831

13. Matanes $\mathrm{E}$, Volodarsky-Perel A, Eisenberg N, et al. Endometrial cancer in germline BRCA mutation carriers: a systematic review and meta-analysis. J Minim Invasive Gynecol 2021;28:947-956.

14. Jiang $X, L i X, L i W$, et al. PARP inhibitors in ovarian cancer: sensitivity prediction and resistance mechanisms. J Cell Mol Med 2019;23:2303-2313.

15. Li H, Liu ZY, Wu N, et al. PARP inhibitor resistance: the underlying mechanisms and clinical implications. Mol Cancer 2020;19:107.

16. Horak P, Weischenfeldt J, von Amsberg G, et al. Response to olaparib in a PALB2 germline mutated prostate cancer and genetic events associated with resistance. Cold Spring Harb Mol Case Stud 2019;5:a003657.

17. Gomez MK, Illuzzi G, Colomer C, et al. Identifying and overcoming mechanisms of PARP inhibitor resistance in homologous recombination repairdeficient and repair-proficient high grade serous ovarian cancer cells. Cancers (Basel) 2020;12:1503.

18. Daly MB, Pilarski R, Yurgelun MB, et al. NCCN Guidelines Insights: Genetic/Familial High-Risk Assessment: Breast, Ovarian, and Pancreatic, Version 1.2020. J Natl Compr Canc Netw 2020;18:380-391.

19. Yang $X$, Leslie G, Doroszuk $A$, et al. Cancer risks associated with germline PALB2 pathogenic variants: an international study of 524 families. J Clin Oncol 2020;38:674-685.

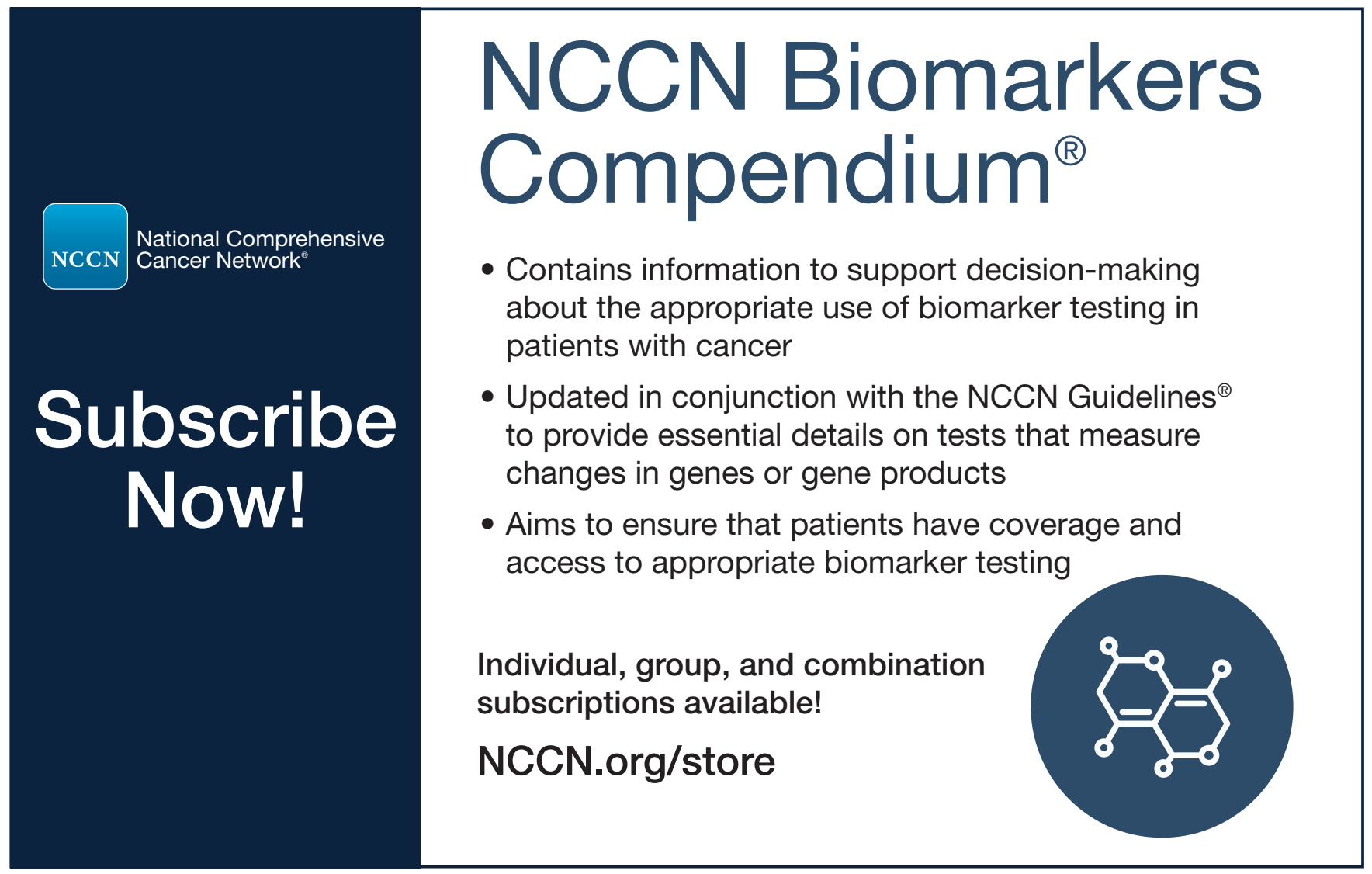

JNCCN-0303-112 